\title{
Surface Silanization of Graphene Oxide Under Mild Reaction Conditions
}

\author{
Tiago Serodre, ${ }^{a}$ Norma A. P. Oliveira, ${ }^{a}$ Douglas R. Miquita, ${ }^{b}$ Max P. Ferreira, ${ }^{a}$ \\ Adelina P. Santos, ${ }^{a}$ Valdirene G. Resende ${ }^{c}$ and Clascídia A. Furtado ${ }^{\circledR} * a$ \\ ${ }^{a}$ Centro de Desenvolvimento da Tecnologia Nuclear (CDTN/CNEN), \\ Universidade Federal de Minas Gerais, 31270-901 Belo Horizonte-MG, Brazil \\ ${ }^{b}$ Centro de Microscopia, Universidade Federal de Minas Gerais, \\ 31270-901 Belo Horizonte-MG, Brazil
}

${ }^{c}$ Centro de Tecnologia de Ferrosos, Vale SA, 34000-000 Nova Lima-MG, Brazil

\begin{abstract}
The functionalization of graphene oxide with organosilanes is an important strategy to yield graphene-silica composites as well as to create nanomaterials for use as reinforcement in polymer nanocomposites and corrosion-inhibiting coatings for metals, among other uses. However, depending on the reaction conditions used, the organosilanes tend to self-condense, encapsulating the graphene oxide in a silica-like layer which can impair its properties by hiding its real surface and two-dimensionality. In this paper we describe a facile route for the functionalization of commercial graphene oxide with (3-aminopropyl) triethoxysilane using low concentrations and mild reaction conditions, and yielding amine and silanol surface-modified graphene nanohybrids while preserving its two-dimensional characteristics. The material obtained was characterized by X-ray photoelectron spectroscopy (XPS), Fourier transform infrared spectroscopy (FTIR), potentiometric titration, thermogravimetric analysis and electronic microscopy, evidencing the covalent and superficial nature of the functionalization.
\end{abstract}

Keywords: graphene oxide, organosilanes, surface modification, 2D materials

\section{Introduction}

Graphene is a two-dimensional carbon nanomaterial with superlative properties such as very high surface area, excellent thermal and electrical conductivity and high mechanical properties, among others. ${ }^{1,2}$ The latter have attracted much interest to using graphene and its derivatives as filler in composites in order to improve their mechanical properties with the addition of very small quantities of the nanomaterial. ${ }^{3,4}$ One of such derivatives, graphene oxide (GO), couples some of the properties of graphene with ease of production and scalability (it can be readily produced by oxidizing natural graphite) $)^{5,6}$ and better dispersion in polar media, owing to the oxygen-containing groups such as hydroxyl, carboxyl and epoxy present in its surface. ${ }^{7}$ The incorporation of GO in polymeric, ${ }^{8-12}$ ceramic $^{13}$ and even metallic matrices ${ }^{14}$ has been widely reported with impressive gains in the mechanical performance of the resulting composites. Another factor that makes GO such

*e-mail: clas@cdtn.br a versatile material is the relative ease with which it can be functionalized to yield different products with fine-tuned properties. The oxygen-containing groups on its surface are relatively reactive and can be coupled with different molecules changing the surface characteristics of the material, while retaining its desirable properties. ${ }^{12,15}$

Graphene oxide has been modified with organosilanes such as (3-aminopropyl) triethoxysilane (APTES), (3-aminopropyl) trimethoxysilane (APTMS) and tetraethoxysilane (TEOS) resulting in $\mathrm{GO}_{-} \mathrm{SiO}_{2}$ hybrids, and these hybrids have been used as a means of improving the mechanical properties of nanocomposites based on epoxy, ${ }^{8,9,12,16,17}$ silica, ${ }^{18}$ polyurethane,,${ }^{11,19}$ chitosan and others. They have also found application enhancing the corrosion protection performance of polymeric coatings for metals, ${ }^{20-22}$ as solid catalysts, ${ }^{23}$ and as anchoring substrates for gold nanoparticles. ${ }^{24}$ Especially for the functionalization of GO with APTES, it is common to explore the carbodiimide route, using, for example, $N, N^{\prime}$-dicyclohexylcarbodiimide (DCC) as a catalyst for the amidation. In many of the cases found in the literature, APTES is used in excess, either as 
a pure liquid or mixed with solvents such as ethanol and toluene, and the reaction is carried out for a relatively long time between 12 and $72 \mathrm{~h}$ at temperatures ranging from 70 to $90{ }^{\circ} \mathrm{C} .18,22$ These conditions cause the APTES to react with the oxygenated groups on the GO surface, grafting the nanomaterial with the organosilane, but also promote the self-condensation and cross-linking of APTES, effectively encapsulating the nanomaterial in a thick $\mathrm{SiO}_{2}$ layer. This encapsulation might be desirable in some cases, because it prevents the graphene oxide surface from being exposed to the environment in which the hybrid is to be used. Sun et al. ${ }^{21}$ for example, encapsulated reduced GO in APTES by reacting it with pure APTES and using DCC as a catalyst. The authors observed that the encapsulation completely inhibited the undesirable corrosion-promotion activity of GO on metals when embedded in a polyvinyl butyral coating. However, in many cases it may be desirable to have only a superficial modification of the GO, creating a hybrid that preserves the two-dimensional characteristics intrinsic to graphene-derived nanomaterials, in such a way that the properties arising from this characteristic can be maintained and explored in dispersion and in its insertion in different media and matrices.

The functionalization of GO with APTES is commonly described as either involving the $\mathrm{OH}$ groups on the surface of the GO, which react with the ethoxysilane groups of the APTES releasing ethanol and forming Si-O-C bonds, ${ }^{16,17,21}$ or involving epoxy groups reacting with the amine of the APTES, creating secondary amines..$^{18,25}$ In both cases, the moieties introduced may react with further APTES molecules, forming a silica-like structure on the surface of the GO. These two possible pathways are shown schematically in Figure 1.

In this work we describe the surface modification of commercially available graphene oxide with APTES, by using much simpler and milder reaction conditions than previously described in the literature and avoiding the use of the toxic substances toluene and DCC, yielding better control of functionalization degree and

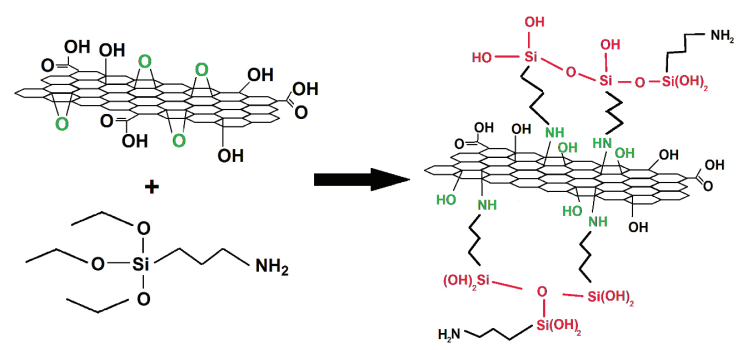

(a) creating a GO/APTES nanohybrid while preserving the two-dimensional characteristics, by avoiding an excessive self-condensation of the APTES on the surface of the GO. We also demonstrate that the covalent attachment of APTES happens simultaneously via both mechanisms shown in Figure 1. The material synthesized may find application as an additive to polymers including epoxy and polyurethane. The materials obtained were characterized by X-ray photoelectron spectroscopy (XPS), Fourier transform infrared spectroscopy (FTIR), thermogravimetric analysis, electronic microscopy and potentiometric titration.

\section{Experimental}

\section{Materials}

Graphite oxide was purchased from Nacional de Grafite Ltda. (Itapecerica, Brazil). In order to reduce its particle size it was ground in a planetary ball mill (Retsch, PM 100) in a $250 \mathrm{~mL}$ stainless steel vase with $10 \mathrm{~mm}$ stainless steel balls during $15 \mathrm{~min}$ at $500 \mathrm{rpm}$ for further use and called gGO. (3-Aminopropyl) triethoxysilane (APTES) was purchased from Sigma-Aldrich (St. Louis, USA) and used as received. All other materials were of analytical grade and available commercially.

\section{Graphite oxide purification}

Graphite oxide was purified in order to remove remaining reagents used in the oxidation as well as oxidation debris, which are very small highly oxidized graphitic particles usually formed during the oxidation of graphite and can negatively affect the final chemical properties of the GO, including its dispersibility and reactivity. ${ }^{26}$ About $10 \mathrm{~g}$ of the ground graphite oxide were first mixed with water, centrifuged at a relative centrifugal force $(\mathrm{RCF})$ of $14,000 \times \mathrm{g}$, washed with a $30 \% \mathrm{HCl}$ solution, then neutralized with a $\mathrm{NaOH}$ solution of $\mathrm{pH} 12$ until a neutral $\mathrm{pH}$ was achieved, which took five washings in

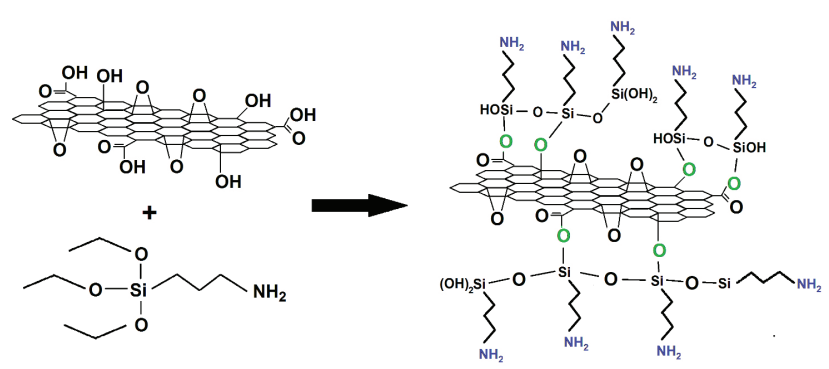

(b)

Figure 1. Schematic representation of the two reaction routes commonly described for the functionalization of GO with APTES: opening of epoxy groups by the APTES amines (a); and OH reacting with the ethoxysilane groups of the APTES (b). 
the centrifuge, again at $14,000 \times \mathrm{g}$. Finally it was washed six times with alternating water and ethanol in the centrifuge. The final material was mixed with diethyl ether and filtered through a $45 \mu \mathrm{m}$ polytetrafluoroethylene (PTFE) membrane and vacuum dried. The resulting material was again ground in a planetary ball mill at the same conditions as described above, and passed through a $297 \mu \mathrm{m}$ sieve. This sample was called pGO.

\section{GO functionalization}

$20 \mathrm{mg}$ of pGO were dispersed in $20 \mathrm{~mL}$ of ethylene glycol with a Silverson L5M-A high shear mixer at $10,000 \mathrm{rpm}$ for $10 \mathrm{~min}$ with a $19 \mathrm{~mm}$ mixing head. After this point, $100 \mu \mathrm{L}$ of APTES were added to the mixture, and the dispersion with the high shear mixer was continued for a further $5 \mathrm{~min}$. Thereafter, the mixture was immediately diluted in acetone, centrifuged and washed five times with acetone, being centrifuged each time at an RCF of $12,000 \times \mathrm{g}$. The final pellet was washed with diethyl ether and vacuum filtered through a PTFE membrane with pore diameter of $45 \mu \mathrm{m}$ and dried in vacuum. This sample was called GO + APTES.

\section{Sample characterization}

Scanning electron microscopy (SEM) images were acquired with a Thermo Fisher Scientific Quanta 200 FEI microscope. The samples were deposited onto a carbon tape and were not metallized.

Transmission electron microscopy (TEM) images were acquired in a Tecnai G2-12, SpiritBiotwin FEI, $120 \mathrm{kV}$ microscope and in a Tecnai G2-20, SuperTwin FEI, $200 \mathrm{kV}$ microscope. The samples were dispersed in water by ultrasonication, and a small droplet of each dispersion was deposited onto a holey carbon TEM grid and vacuum dried.

Potentiometric titrations were carried out in a Metrohm Tritano automatic titrator with $0.018 \mathrm{~mol} \mathrm{~L}^{-1} \mathrm{NaOH}$ and $0.001 \mathrm{~mol} \mathrm{~L}^{-1} \mathrm{HCl}$ solutions. The samples were dispersed in deionized water by ultrasonication at a concentration of $0.1 \mathrm{mg} \mathrm{mL}^{-1}$.

Zeta potential measurements were carried out in a Malvern Zetasizer Nano at $25{ }^{\circ} \mathrm{C}$. The samples were dispersed at a concentration of $0.1 \mathrm{mg} \mathrm{mL}^{-1}$ in a $2 \mathrm{mmol} \mathrm{L}^{-1}$ potassium chloride solution by ultrasonication.

FTIR spectra were obtained with a Thermo Scientific Nicolet 6700 spectrophotometer. The samples were mixed with $\mathrm{KBr}$ and pressed for the analysis. The main peaks were fitted using Voigt profile curves for the absorbance plot of the spectra. XPS analyses were performed with a Thermo Scientific K-Alpha spectrometer with aluminium $\mathrm{K} \alpha$ radiation. The samples were deposited onto a copper tape. Spectral data was treated using the software CasaXPS. ${ }^{27}$

CHN elemental analysis was done in a PerkinElmer 2400 Series II CHNS/O analyzer, with about $1 \mathrm{mg}$ of each sample.

Conventional thermogravimetric analysis (TGA) and differential thermogravimetry (DTG) for the determination of water content in the samples was performed in a TA instruments SDT-6500 equipment. About $10 \mathrm{mg}$ of each sample were heated in an alumina crucible from room temperature to $1000{ }^{\circ} \mathrm{C}$ at a rate of

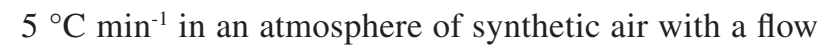
rate of $100 \mathrm{~mL} \mathrm{~min}^{-1}$.

Stepwise isothermal thermogravimetric analysis was performed in a TA Instruments Q5000 equipment. About $10 \mathrm{mg}$ of each sample were heated in an alumina crucible from room temperature to $1000{ }^{\circ} \mathrm{C}$ at a rate of $5{ }^{\circ} \mathrm{C} \mathrm{min}^{-1}$ in an inert atmosphere of nitrogen with a flow rate of $25 \mathrm{~mL} \mathrm{~min}^{-1}$. The weight loss threshold for entering isothermal steps was $0.50 \% \mathrm{~min}^{-1}$, the threshold for exiting isothermal steps was $0.05 \% \mathrm{~min}^{-1}$.

Hyphenated thermogravimetric analysis and Fourier transform infrared spectroscopy (TGA-FTIR) was performed in a PerkinElmer STA 8000 simultaneous thermal analyzer coupled with a Frontier FTIR spectrophotometer. About $30 \mathrm{mg}$ of each sample were heated in an alumina crucible from room temperature to $750{ }^{\circ} \mathrm{C}$ at a rate of $20{ }^{\circ} \mathrm{C} \mathrm{min}{ }^{-1}$ in an inert atmosphere of helium with a flow rate of $30 \mathrm{~mL} \mathrm{~min}^{-1}$.

\section{Results and Discussion}

\section{$\mathrm{CHN}$ and XPS analysis}

The survey XPS spectra of the samples gGO, pGO and $\mathrm{GO}+$ APTES are presented in Figure 2, and the elemental composition obtained from the analysis of these spectra is shown in Table 1. The sample gGO contains $2.1 \%$ of sulfur atoms and $0.7 \%$ of nitrogen atoms, thought to be a residue of sulfuric and nitric acid used during the oxidation of graphite, which are not present in the other samples, showing that the purification step was able to remove this contaminant. Nevertheless, the purification introduced a small amount of sodium atoms (not shown in Figure 2) from the $\mathrm{NaOH}$ used during the basic washing steps. The relative oxygen content of the sample pGO decreases compared to the sample gGO, signaling the removal of highly oxidized particles adsorbed on the surface commonly called oxidation debris. The removal of these debris is an important step prior to the functionalization of oxidized carbon nanomaterials such as GO, because they 
hide the surface of the nanomaterial, ${ }^{28}$ and, being highly oxidized, may react preferentially with the APTES. The GO + APTES hybrid contains $4.0 \%$ of silicon and $2.6 \%$ of nitrogen, consistent with a superficial functionalization of GO with APTES. These results agree well with the elemental composition obtained from $\mathrm{CHN}$ analysis (Table 2). Considering that XPS is a superficial technique, whereas $\mathrm{CHN}$ is a bulk technique, similar results from both techniques provide an indication that the samples have very high aspect ratio.

The high resolution $\mathrm{C} 1 \mathrm{~s}$ spectra of both $\mathrm{pGO}$ and GO + APTES samples (Figure 3) give information about the chemical bonding of carbon atoms on the surface of the samples. The relative contributions of each bond assigned in the spectra are summarized in Table 3. Both samples show an intense peak around $284.6 \mathrm{eV}$ corresponding to $\mathrm{C}-\mathrm{C}$ bonds, which can be further divided into $\mathrm{sp}^{3}$ and $\mathrm{sp}^{2}$ contributions resulting in a better fit. At higher binding energies, we observe the contributions of the oxygenated $\mathrm{C}$ atoms. In the pGO spectrum, we find an intense peak around $287.0 \mathrm{eV}$ corresponding to $\mathrm{C}-\mathrm{O}$ carbons in functional groups such as hydroxyl and epoxy, whereas the corresponding peak in the GO + APTES spectrum is considerably less intense, evidencing the covalent modification of the GO sheets and suggesting that the functionalization reaction involves these groups. It has been shown that the reaction of GO with molecules containing primary amines takes place with the opening of epoxy groups on the basal plane of the GO, rather than with the formation of amides from the carboxylic groups. ${ }^{29}$ Yang et al. ${ }^{18}$ proposed that the APTES amine groups react with the epoxy groups on the surface of the graphene oxide creating secondary amines, which would be consistent with the reduction in the intensity of this peak. Furthermore, a peak appears at around $286.4 \mathrm{eV}$ in the spectrum of the functionalized sample, corresponding to carbons involved in $\mathrm{C}-\mathrm{N}$ bonds. The contributions of more oxidized carbons, involved in $\mathrm{C}=\mathrm{O}$ bonds and carboxyl groups, can be seen at 287.9 and $289.0 \mathrm{eV}$, respectively, and at even higher energies, we see the broad shake-up features caused by $\pi-\pi^{*}$ transitions in the $\mathrm{sp}^{2}$ carbons. These oxygenated groups are also slightly reduced in the functionalized sample in comparison with its precursor.

The high-resolution $\mathrm{N}$ 1s spectrum of sample $\mathrm{GO}+$ APTES (Figure 4a) can be deconvoluted into two peaks. The lower binding energy peak centered at $399.8 \mathrm{eV}$ was assigned to $\mathrm{N}$ atoms participating in secondary amine bonds ${ }^{30}$ and contributes with $43 \%$ of $\mathrm{N} 1 \mathrm{~s}$ peak area,
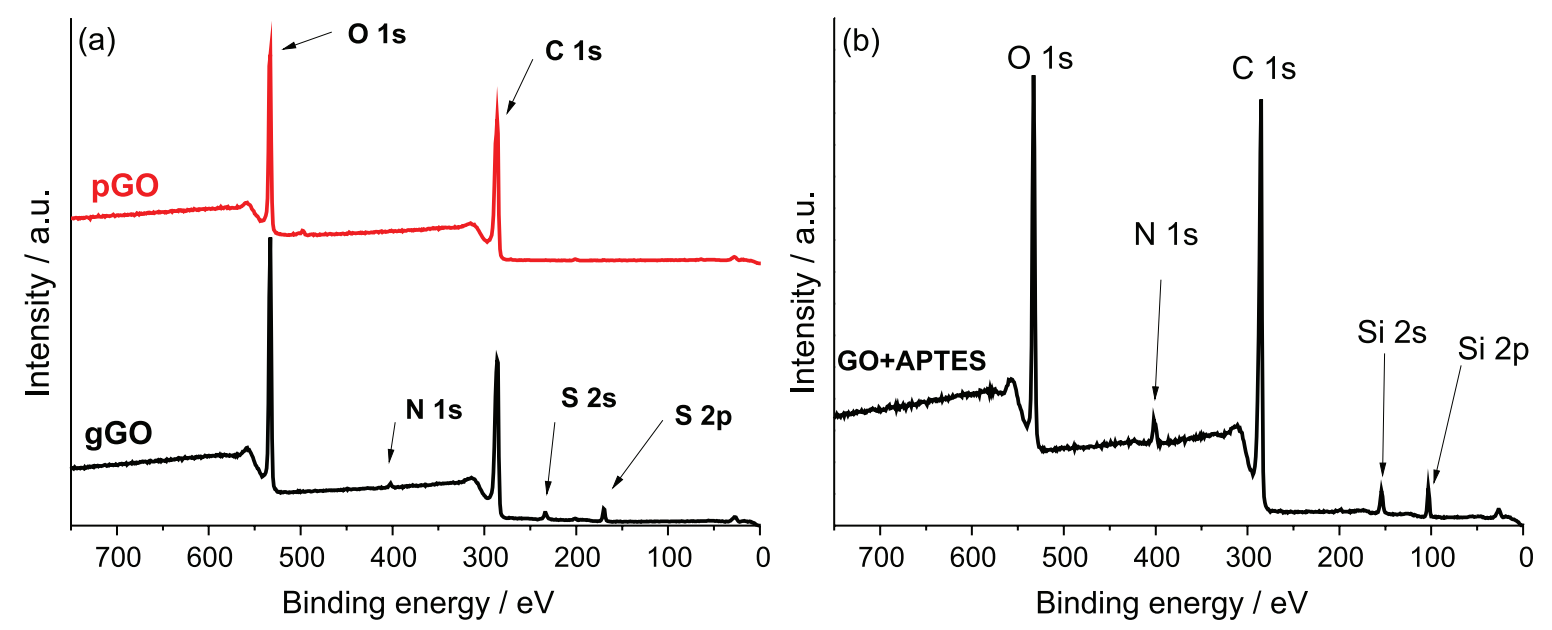

Figure 2. Survey XPS spectra for samples (a) gGO and pGO and (b) GO + APTES.

Table 1. Elemental composition of the nanomaterials as determined by XPS in atomic ratio

\begin{tabular}{lcccccc}
\hline \multirow{2}{*}{ Sample } & \multicolumn{6}{c}{ Element / at. } \\
\cline { 2 - 7 } & $\mathrm{C}$ & $\mathrm{O}$ & $\mathrm{S}$ & $\mathrm{Na}$ & $\mathrm{N}$ & $\mathrm{Si}$ \\
\hline $\mathrm{gGO}$ & 69.3 & 28.0 & 2.1 & 0 & 0.7 & 0 \\
$\mathrm{pGO}$ & 74.2 & 25.4 & 0 & 0.4 & 0 & 0 \\
GO + APTES & 71.3 & 22.2 & 0 & 0 & 2.6 & 4.0 \\
\hline
\end{tabular}

gGO: ground graphite oxide; pGO: purified graphite oxide; APTES: (3-aminopropyl) triethoxysilane.
Table 2. CHN elemental analysis of the nanomaterials in weight percentage

\begin{tabular}{lccc}
\hline \multirow{2}{*}{ Sample } & \multicolumn{3}{c}{ Element / wt. $\%$} \\
\cline { 2 - 4 } & $\mathrm{C}$ & $\mathrm{H}$ & $\mathrm{N}$ \\
\hline gGO & 65.0 & 3.6 & 0.1 \\
pGO & 71.8 & 3.6 & 0.1 \\
GO + APTES & 66.0 & 6.0 & 4.7 \\
\hline
\end{tabular}

gGO: ground graphite oxide; pGO: purified graphite oxide; APTES: (3-aminopropyl) triethoxysilane. 

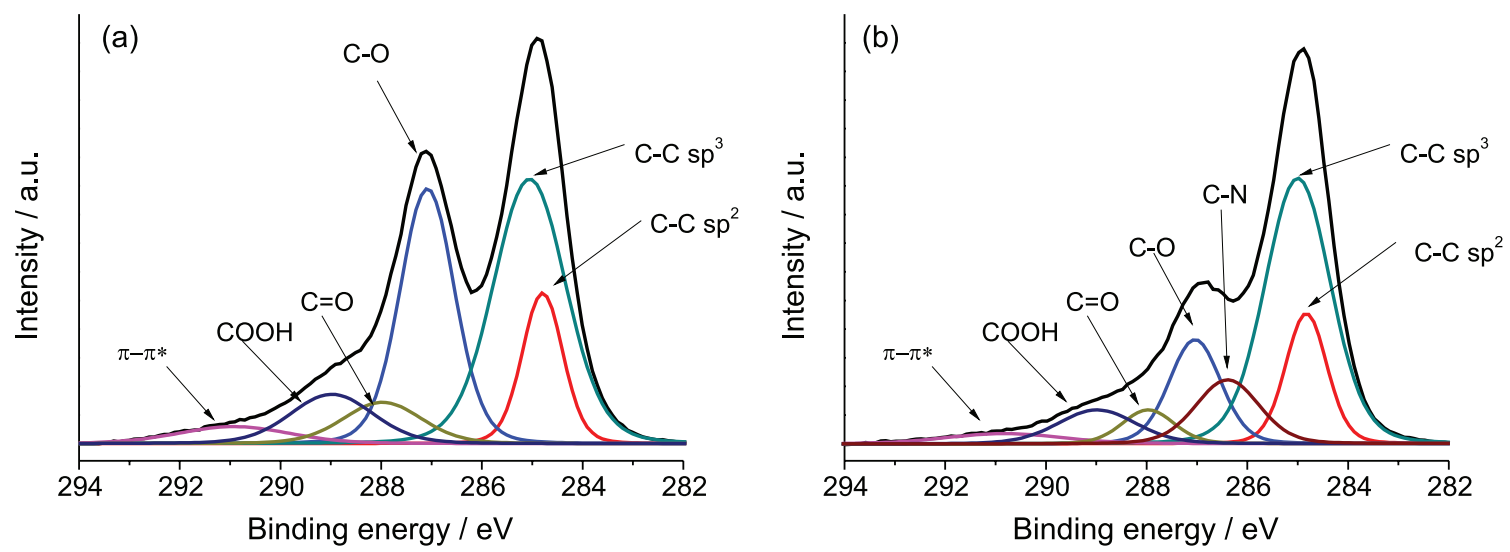

Figure 3. High-resolution $\mathrm{C}$ 1s spectrum of (a) pGO and (b) GO + APTES.

Table 3. Relative contribution of bonds identified from the high resolution C 1s XPS spectra

\begin{tabular}{lcccccc}
\hline & \multicolumn{5}{c}{ Relative contribution $/ \%$} \\
\cline { 2 - 7 } Sample & $\begin{array}{c}\mathrm{C}-\mathrm{C} \mathrm{sp} \\
284.6 \mathrm{eV}^{\mathrm{a}}\end{array}$ & $\begin{array}{c}\mathrm{C}-\mathrm{C} \mathrm{sp} \\
284.8 \mathrm{eV}^{\mathrm{a}}\end{array}$ & $\begin{array}{c}\mathrm{C}-\mathrm{N} \\
286.4 \mathrm{eV}^{\mathrm{a}}\end{array}$ & $\begin{array}{c}\mathrm{C}-\mathrm{O} \\
287.0 \mathrm{eV}^{\mathrm{a}}\end{array}$ & $\begin{array}{c}\mathrm{C}=\mathrm{O} \\
287.9 \mathrm{eV}^{\mathrm{a}}\end{array}$ & $\begin{array}{c}\mathrm{COOH} \\
289.0 \mathrm{eV}^{\mathrm{a}}\end{array}$ \\
\hline pGO & 13 & 41 & 0 & 30 & 7 & 9 \\
GO + APTES & 15 & 47 & 12 & 14 & 5 & 7 \\
\hline
\end{tabular}

${ }^{a}$ Assigned bond and corresponding binding energy. pGO: purified graphite oxide; APTES: (3-aminopropyl) triethoxysilane.

corroborating the formation of secondary amines from GO epoxy groups, and APTES primary amines. The other peak at $401.8 \mathrm{eV}$, corresponding to protonated primary amines, ${ }^{31,32}$ suggests that while most of the amines of APTES react with epoxy groups, a considerable amount does not, and continue as primary amines in the GO-APTES hybrid, arising from APTES molecules bound by its silane groups.

Finally, the silicon S 2p spectrum (Figure 4b) shows a peak around $103 \mathrm{eV}$, which is consistent with silicon atoms participating in $\mathrm{Si}-\mathrm{O}-\mathrm{Si}$ and $\mathrm{Si}-\mathrm{O}-\mathrm{C}$ bonds. ${ }^{33}$ The peak was deconvoluted into two contributions to account for the spin-orbit splitting of the $\mathrm{p}$ orbital.

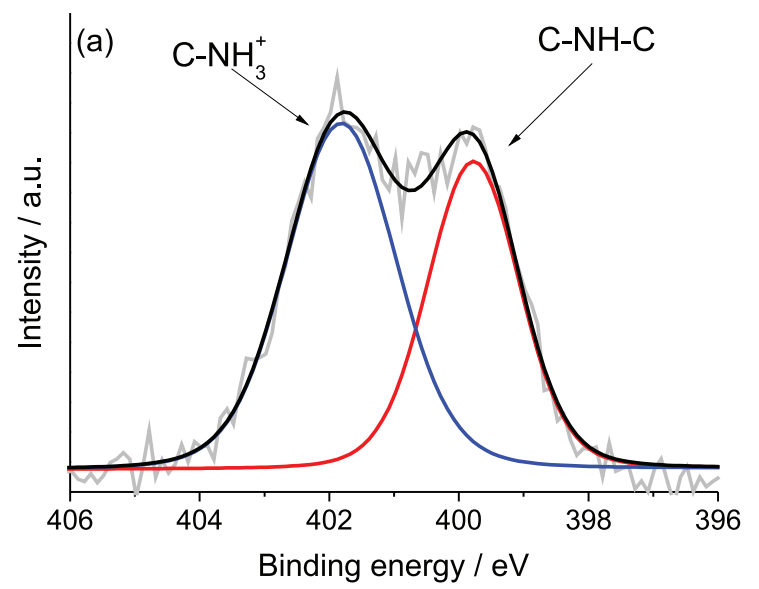

\section{FTIR analysis}

The FTIR spectra of the samples gGO, pGO and $\mathrm{GO}+$ APTES are presented in Figure 5. In Figure 6, the region between 1800 and $800 \mathrm{~cm}^{-1}$ where many interesting spectral features overlap, is shown in greater detail. The spectra of samples pGO and GO + APTES had the main peaks deconvoluted for better clarity and to facilitate the identification of absorption bands. The spectrum of the starting material, gGO, displays absorption bands characteristic of the oxygen-containing groups present in graphene oxide, of which we have highlighted the following: hydroxyl groups show characteristic bands at

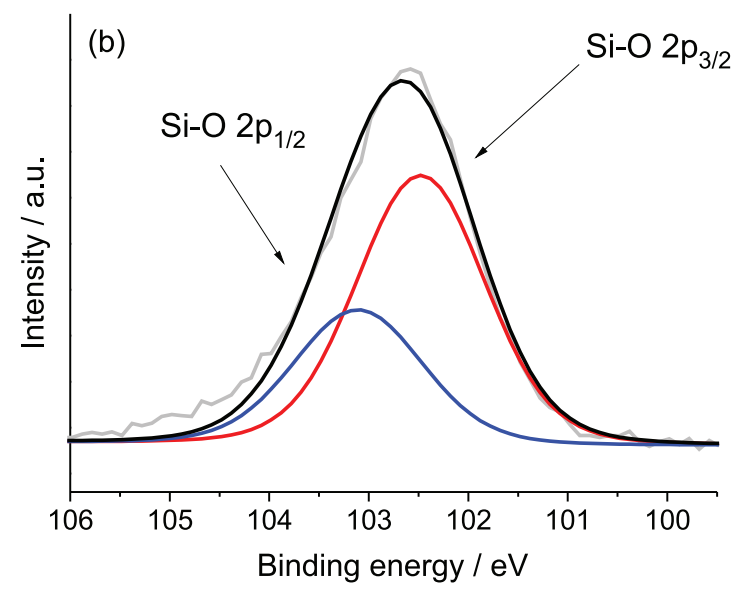

Figure 4. (a) High-resolution N 1s spectrum of sample GO + APTES; (b) high-resolution Si 2p spectrum of sample GO + APTES. 
3370,1380 and $1060 \mathrm{~cm}^{-1}$ corresponding to the stretching of $\mathrm{O}-\mathrm{H}$ bonds, $\mathrm{O}-\mathrm{H}$ bending and $\mathrm{C}-\mathrm{O}$ stretching, respectively. The presence of carboxylic acid groups is evidenced by an absorption band at ca. $3200 \mathrm{~cm}^{-1}$, associated to the stretching of $\mathrm{O}-\mathrm{H}$ bonds, as well as a band at $1716 \mathrm{~cm}^{-1}$, corresponding to the stretching of $\mathrm{C}=\mathrm{O}$ bonds. At $1232 \mathrm{~cm}^{-1}$, the absorption is associated with $\mathrm{C}-\mathrm{O}-\mathrm{C}$ stretching of epoxy groups. Finally the band at $1590 \mathrm{~cm}^{-1}$ corresponds to the stretching of $\mathrm{C}=\mathrm{C}$ bonds in the graphitic skeleton. ${ }^{34-36}$ Sample pGO shows bands in approximately the same wavenumbers as gGO; however, the relative intensity of the hydroxyl and carboxyl groups in the purified sample is reduced, evidencing the removal of highly oxidized debris from the surface of the GO.

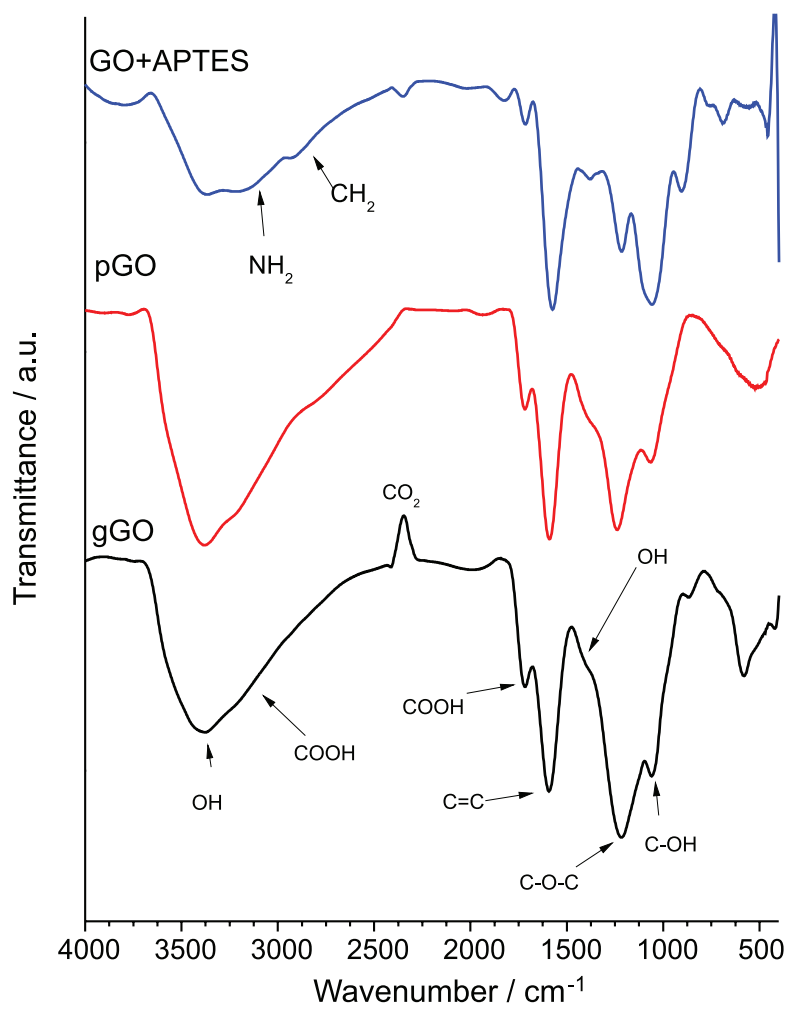

Figure 5. FTIR spectra $(\mathrm{KBr})$ of the samples gGO, pGO and GO + APTES. Peaks appearing at around $2345 \mathrm{~cm}^{-1}$ are due to atmospheric $\mathrm{CO}_{2}$.

In the functionalized sample, GO + APTES, it is still possible to see the hydroxyl and carboxyl bands at approximately the wavenumbers described above, although their relative intensity is greatly diminished, signifying that these groups were largely consumed during the functionalization reactions. Furthermore, new absorption bands appeared: in Figure 5 we highlighted those at $3180 \mathrm{~cm}^{-1}$, corresponding to the $\mathrm{N}-\mathrm{H}$ stretching in amine and at $2890 \mathrm{~cm}^{-1}$, corresponding to $\mathrm{C}-\mathrm{H}$ stretching in aliphatic $\mathrm{CH}_{2}$ groups. In Figure 6, we also see new bands at 894,1014 and $1117 \mathrm{~cm}^{-1}$ associated with stretching of

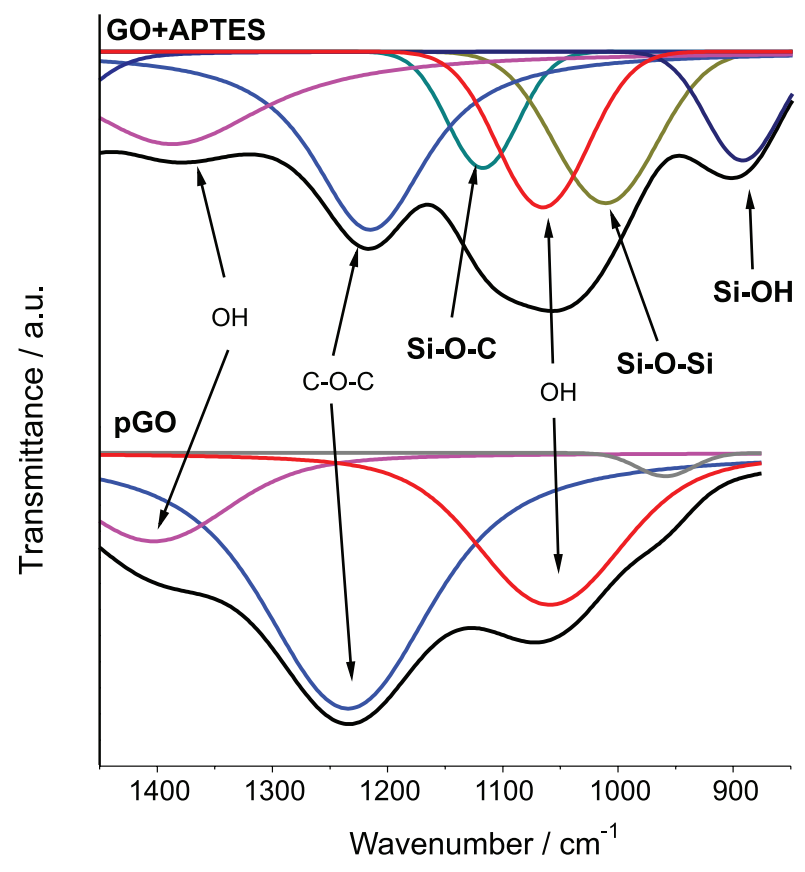

Figure 6. Excerpt of the FTIR spectra $(\mathrm{KBr})$ of the samples pGO and $\mathrm{GO}+\mathrm{APTES}$ with main peaks deconvoluted.

$\mathrm{Si}-\mathrm{O}-\mathrm{Si}, \mathrm{Si}-\mathrm{O}-\mathrm{C}$ and $\mathrm{Si}-\mathrm{OH}$ bands, respectively. ${ }^{8,37-39}$ The reduction of carboxyl, hydroxyl and epoxy groups in the functionalized sample is consistent with the evidence provided by the XPS analysis, and indicates that these groups participate in the reaction with APTES.

\section{Potentiometric titration and zeta potential}

Further evidence for the covalent modification of $\mathrm{GO}$ is given by the potentiometric titration, as shown in Table 4 . The purified GO contains oxygenated groups with $\mathrm{p} K_{\mathrm{a}}$ ranging from 4.5 to 10.0. The most acid groups with $\mathrm{p} K_{\mathrm{a}}$ lower than 6.5 are identified as carboxylic acids. The ones with $\mathrm{p} K_{\mathrm{a}}$ around neutral are associated with lactones and other carbonyl-containing groups. Above a $\mathrm{p} K_{\mathrm{a}}$ of around 8 , there are hydroxyl groups, including phenolic hydroxyls. ${ }^{40-43}$ We observe that while the purified GO has about $3.3 \mathrm{mmol} \mathrm{g}^{-1}$ of acid and basic groups on its surface, this number is reduced to $0.7 \mathrm{mmol} \mathrm{g}^{-1}$ after the functionalization. The amount of acid groups with $\mathrm{p} K_{\mathrm{a}}$ less than 6.5 is reduced by one third in the GO + APTES, and the phenolic groups show an even sharper decrease. $\mathrm{GO}+$ APTES contains about $0.5 \mathrm{mmol} \mathrm{g} \mathrm{g}^{-1}$ of groups with $\mathrm{p} K_{\mathrm{a}}$ greater than 8.0, and this figure accounts for non-reacted hydroxyls from the starting pGO, but may also be due to silanol groups introduced by the functionalization.

It is important to notice that the experimental conditions used for the potentiometric titration do not allow for the identification of amines due to their high expected $\mathrm{p} K_{\mathrm{a}}$. 
Table 4. Amount of groups in samples pGO and GO + APTES in aqueous solution as determined by potentiometric titration

\begin{tabular}{lccc}
\hline & \multicolumn{3}{c}{ Number of groups $/\left(\mathrm{mmol} \mathrm{g}^{-1}\right)$} \\
\hline $\mathrm{p} K_{\mathrm{a}}$ & $4.5-6.5$ & $6.5-8.0$ & $8.0-10.5$ \\
Associated group & $\mathrm{COOH}$ & lactones & $\mathrm{OH}$ \\
$\mathrm{pGO}$ & 0.7 & 0.4 & 2.2 \\
GO + APTES & 0.2 & 0.0 & 0.5 \\
\hline
\end{tabular}

pGO: purified graphite oxide; APTES: (3-aminopropyl) triethoxysilane.

Despite the reduction in the number of polar groups on the surface of the functionalized GO, it can still be dispersed in water by sonication yielding stable dispersions, similarly to the purified GO. The zeta potential of dispersed samples measured in a $\mathrm{KCl}$ solution were $-23.4 \pm 0.9$ and $-19.3 \pm 1.2 \mathrm{mV}$ for the $\mathrm{pGO}$ and the GO + APTES, respectively. The increase in the potential can be explained by the introduction of positively charged $\mathrm{NH}_{3}^{+}$groups on the surface of the functionalized material in neutral $\mathrm{pH}$. However, the absolute value of the zeta potential of GO + APTES is still high enough to explain its stability in aqueous solution.

\section{Thermogravimetric analysis}

Water content in samples pGO was determined by conventional thermogravimetric analysis (curves not shown) to be around $21 \%$ in mass. This water can promote

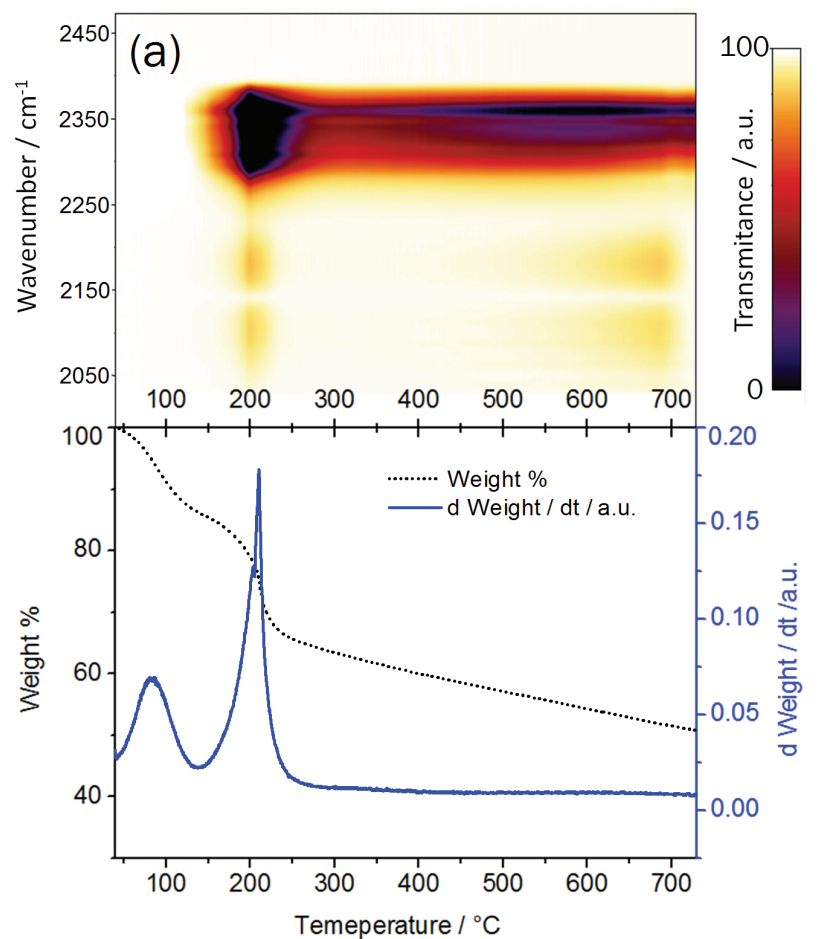

the hydrolysis of the APTES molecules, and therefore, the low concentration of APTES in the reaction medium is important to keep the APTES from self-condensing to a great extent.

Hyphenated thermogravimetric and Fourier transform infrared spectroscopy (TGA-FTIR) analyses of samples pGO and GO + APTES are presented in Figure 7 below. The three-dimensional FTIR spectra show the transmittance of the decomposition products in the region between 2000 and $2500 \mathrm{~cm}^{-1}$, varying with the temperature of decomposition. In this region, it is possible to observe bands corresponding to the two main decomposition products of the samples, namely $\mathrm{CO}_{2}$, with a very intense band around $2350 \mathrm{~cm}^{-1}$, and $\mathrm{CO}$, with $\mathrm{P}$ and $\mathrm{R}$ branches characteristic of diatomic molecules centered at around 2120 and $2175 \mathrm{~cm}^{-1}$, respectively. The information obtained by TGA-FTIR can be compared with the stepwise isothermal TGA curves shown in Figure 8 in order to describe the changes in the surface composition of the GO after the functionalization. It is important to note that the temperature of an event measured by the stepwise technique will correspond approximately to the onset temperature of the same event in conventional TGA, and therefore, the peaks will appear slightly shifted to lower temperatures when comparing Figures 7 and 8 .

It is known that the thermal decomposition of oxygencontaining carbon materials in the range temperatures studied here is due to the decomposition of the surface

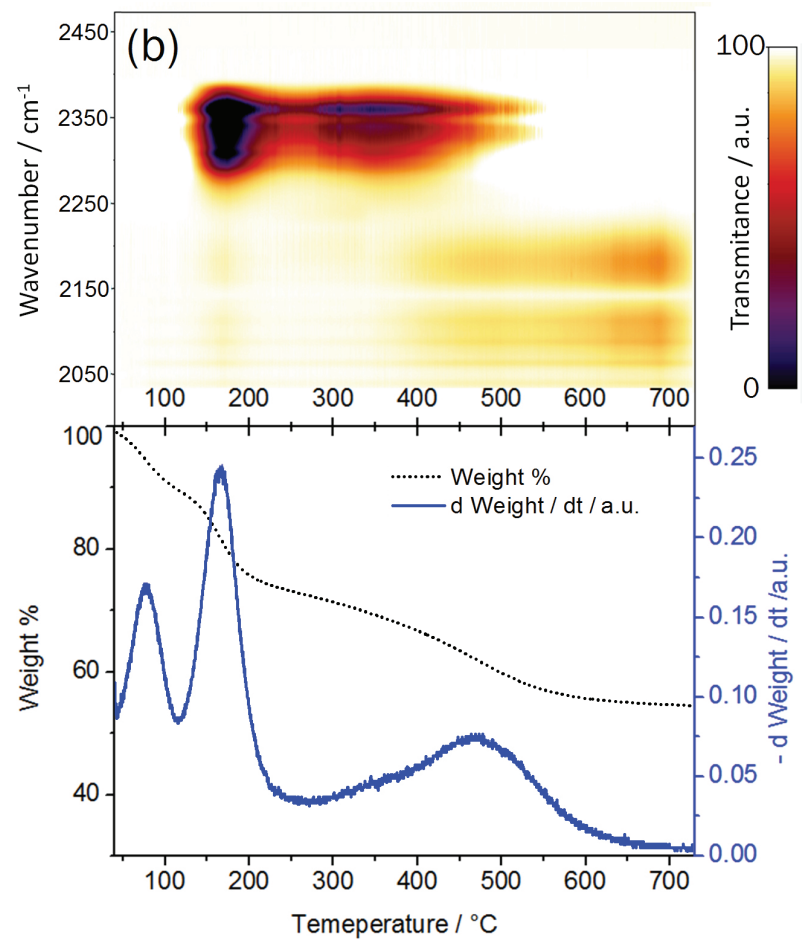

Figure 7. TGA-FTIR curves obtained for samples pGO (a) and GO + APTES (b). 

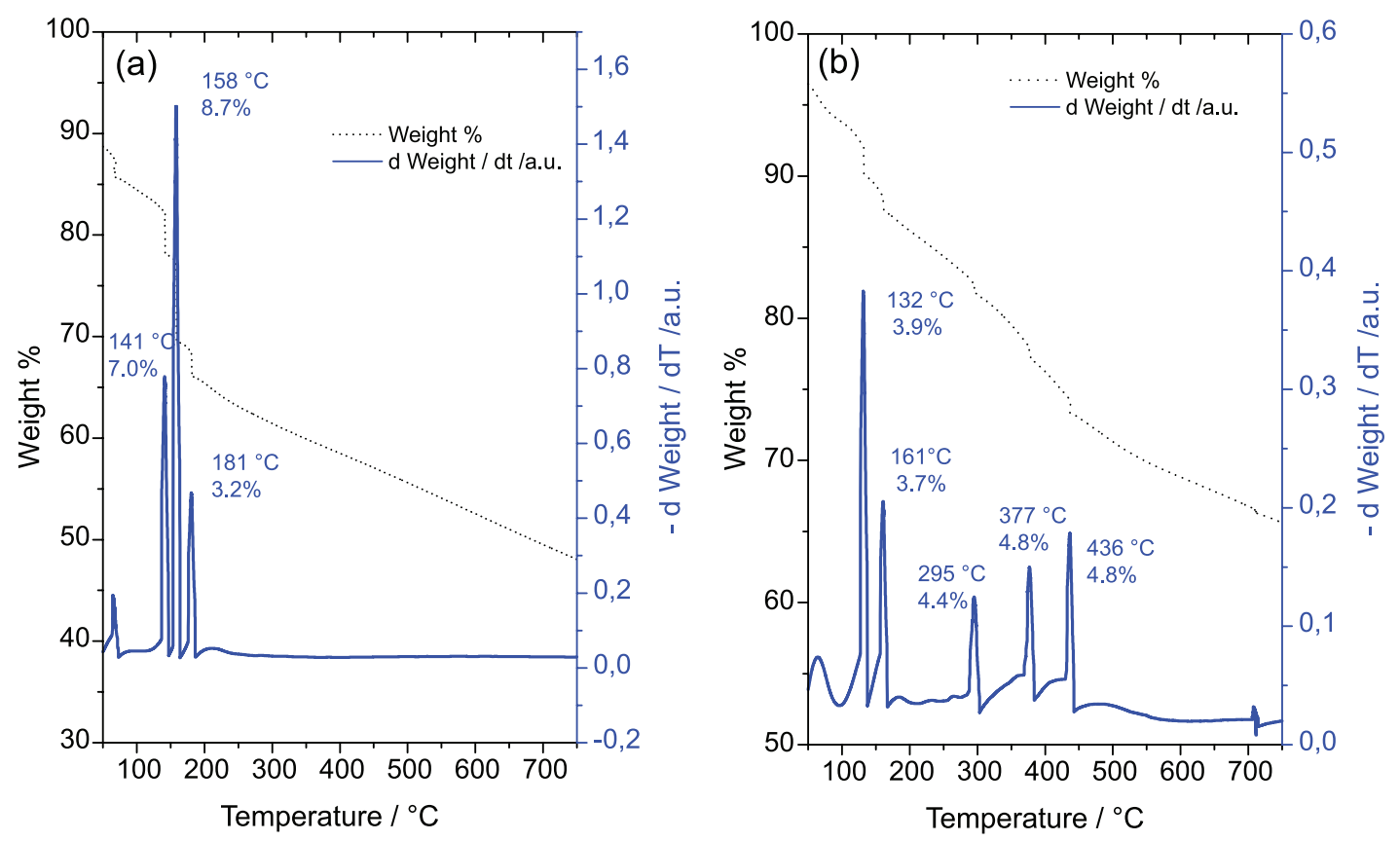

Figure 8. Stepwise isothermal TGA and DTG curves obtained for samples pGO (a) and GO + APTES (b).

oxygenated groups, which can occur with the liberation of either $\mathrm{CO}$ or $\mathrm{CO}_{2}$. As the temperature increases, groups which decompose into $\mathrm{CO}$ will be released roughly in this order: epoxy, ${ }^{44}$ anhydrides; phenols; and carbonyls, while groups which give off $\mathrm{CO}_{2}$ will follow this order: more acidic carboxylic acids; less acidic carboxylic acids; lactones; and peroxides. ${ }^{45-47}$ In Figure 8, one can see that the pGO shows three important events between 120 and $200{ }^{\circ} \mathrm{C}$, which are accompanied by the release of both $\mathrm{CO}$ and $\mathrm{CO}_{2}$, and account for $18.9 \%$ of the mass of the sample. In the same region, for the sample GO + APTES, there are only two major events and they account for only $7.8 \%$ of the samples mass, indicating that oxygenated groups have been consumed during the reaction. Importantly, the release of carbon monoxide in this lower temperatures is greatly diminished in the functionalized sample, suggesting the consumption of epoxy groups, since they are one of the few candidates which decompose into $\mathrm{CO}$ in these temperatures. For higher temperatures, sample pGO decomposes in a relatively constant rate, without major events, whereas the functionalized GO + APTES shows three new events at 295,377 and $436^{\circ} \mathrm{C}$, which are due to the oxygen and nitrogen containing moieties introduced by the APTES. At even higher temperatures, there is a noticeable change in the composition of the decomposition products, with pGO releasing both $\mathrm{CO}$ and $\mathrm{CO}_{2}$ above $600{ }^{\circ} \mathrm{C}$, while $\mathrm{GO}+\mathrm{APTES}$ shows very little carbon dioxide above that temperature.

In accordance with the techniques shown previously, TGA corroborates the fact that the oxygenated groups on the surface of the GO are being consumed by the functionalization reaction, showing that the modification is covalent in nature. TGA-FITR gives strong evidence specifically for the consumption of epoxy groups, together with other oxygenated groups, and, when compared with the evidence provided by potentiometric titration, FTIR and XPS, it is possible to see a trend of the amount of epoxy and $\mathrm{OH}$ groups decreasing at the same time as secondary amines and $\mathrm{Si}-\mathrm{O}-\mathrm{C}$ groups are being formed. Therefore, we propose that both reactions shown in Figure 1 happen simultaneously during the functionalization of GO and APTES in the mild conditions described here, giving rise to a hybrid containing both amines and silanols on its surface, as shown in Figure 9.

\section{Electronic microscopy}

Scanning electronic microscopy (SEM) images of the dried samples gGO, pGO and GO + APTES (Figure 10) show similar morphologies for the three samples: large lamellar grains with tens of micrometers in size, formed by the packaging of the thin sheets with relatively flat surfaces, on top of which one can see structures that appear to be peeling off. Sample GO + APTES does not show a coating of amorphous material suggesting that the functionalization took place on and was limited to the surface of the nanomaterial, without a great degree of self-condensation of the APTES.

Figure 11 shows TEM images of sample pGO. A thin sheet can be seen (Figure 11a) with the typical two 

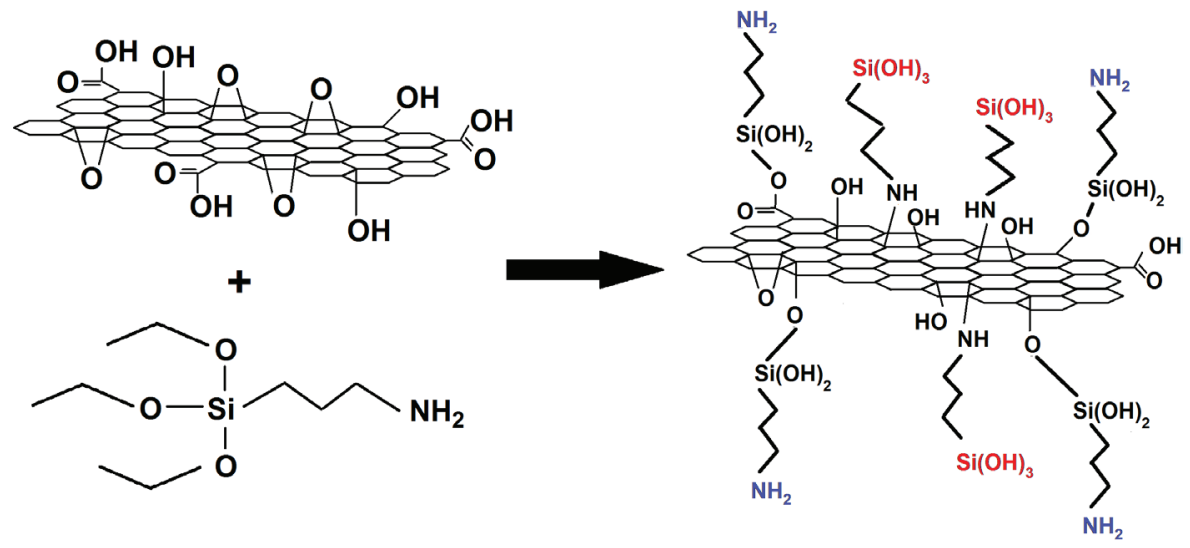

Figure 9. The opening of epoxy groups by the APTES amines and the reaction of OH groups with the ethoxysilane of the APTES take place simultaneously.
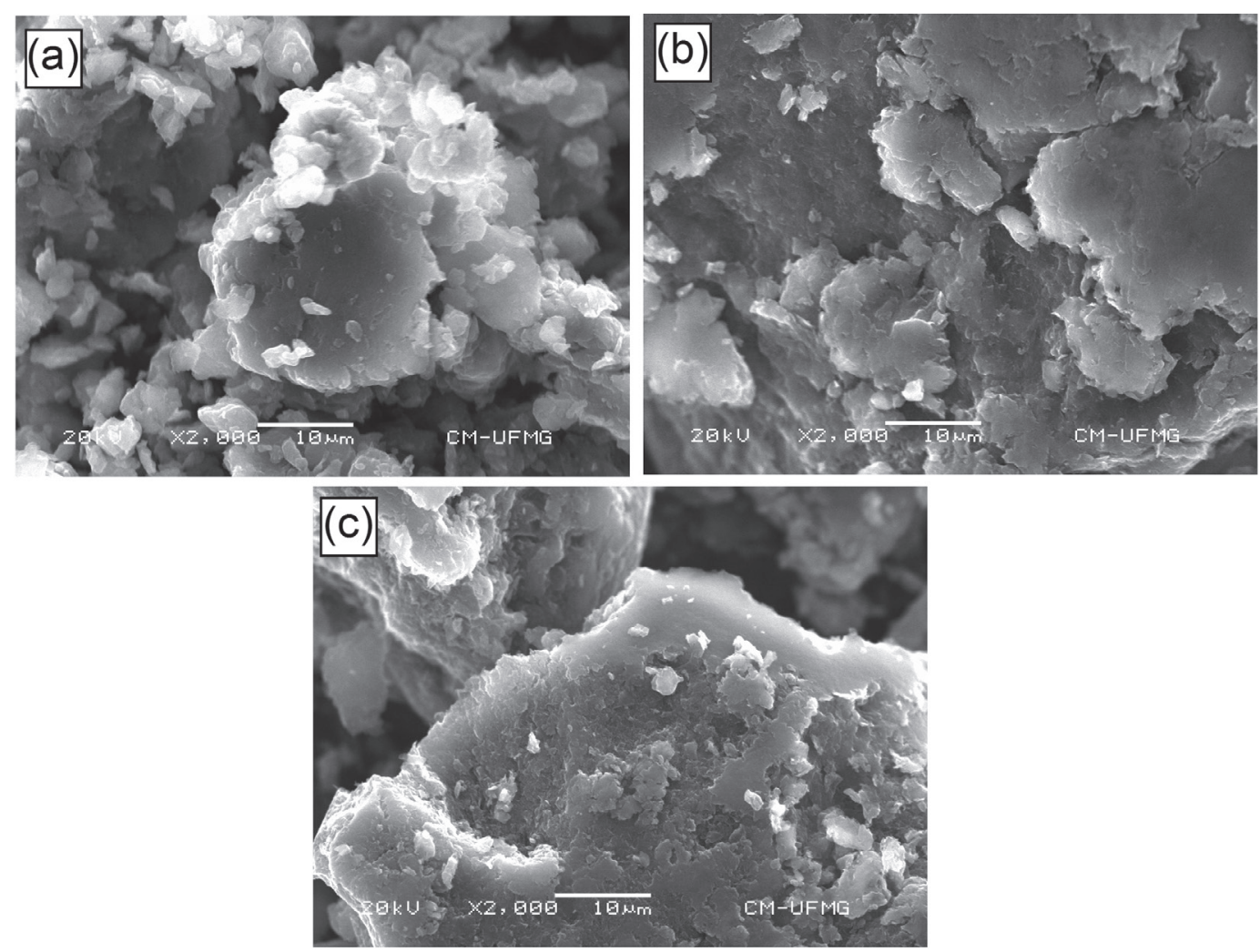

Figure 10. SEM images of samples gGO (a); pGO (b) and GO + APTES (c).

dimensional morphology of GO sheets, with visible layers and the sample folding on itself creating creases. Figures $11 \mathrm{~b}$ and $11 \mathrm{c}$ show details of the region indicated by the arrow in Figure 11a, revealing the stacking of the graphene sheets and the folds in more detail. The sheet shown in Figure 11d is smaller in its lateral size, but also shows the typical morphology of GO sheets mentioned above.

In Figure 12, TEM images of sample GO + APTES are shown. Similar features as pointed out for the pGO can be seen, with a large sheet (Figure 12a) presenting visibly distinct wrinkles and folds creating creases in the material, which can be better observed in the magnified image (Figure 12b). Figure 12c shows a high resolution image of the edge of the fold shown in Figure 12b. In this image, it is possible to see the edge of some sheets (indicated by the arrow) embedded in a surface with a strongly amorphous character. The presence of this amorphous material evidences that the functionalization took place. Many sheets smaller in lateral size are deposited on top of this large one and can be seen throughout Figure 12a. Sample GO + APTES appears to be coated by a very thin layer of material, making it appear thicker and for the edges of distinct layers to appear less sharp when compared to 

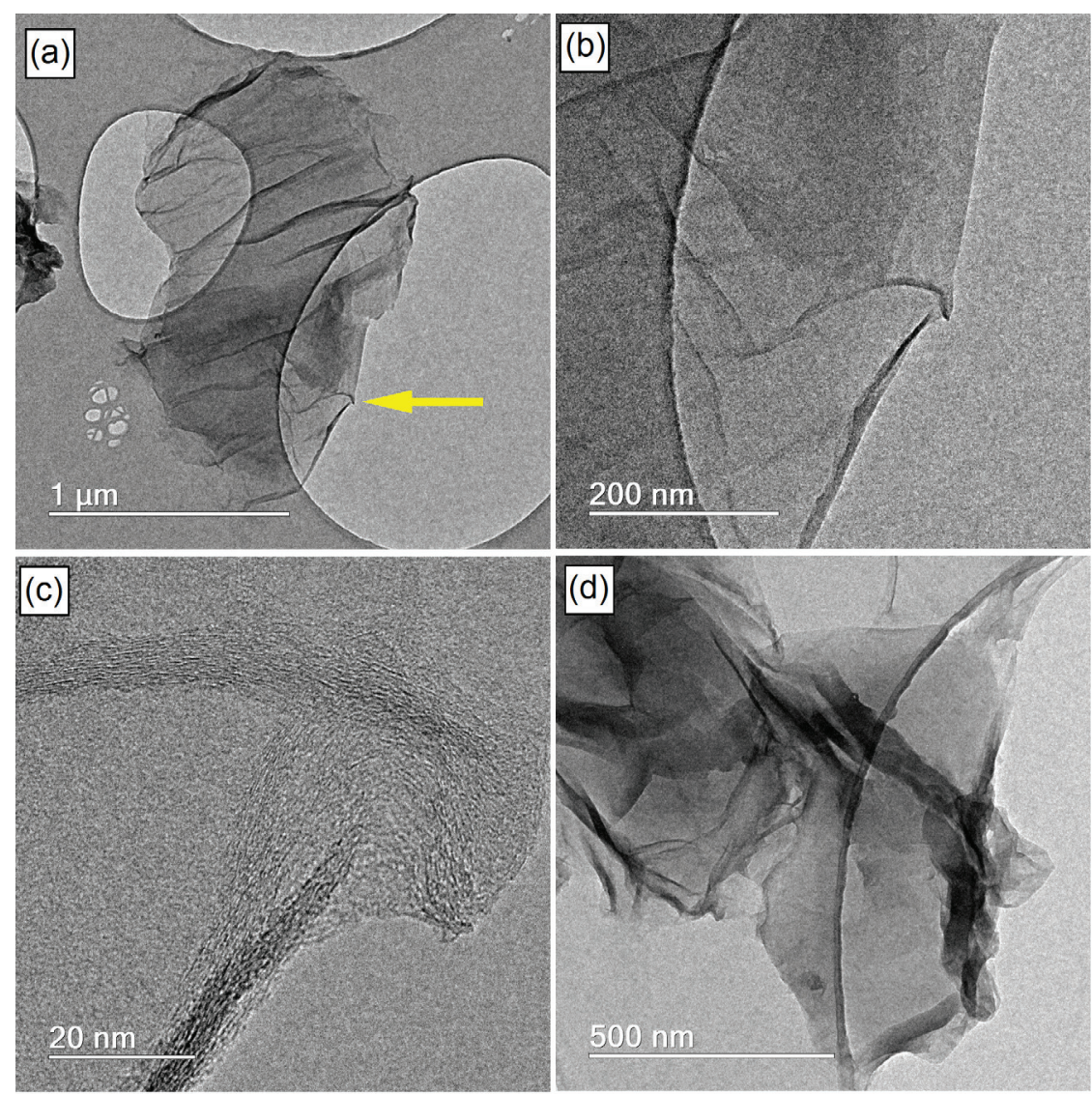

Figure 11. TEM images of sample pGO. (a) A thin sheet of graphene oxide; (b) detail of the region indicated by the arrow; (c) same region at a higher magnification; (d) a smaller sheet of graphene oxide.
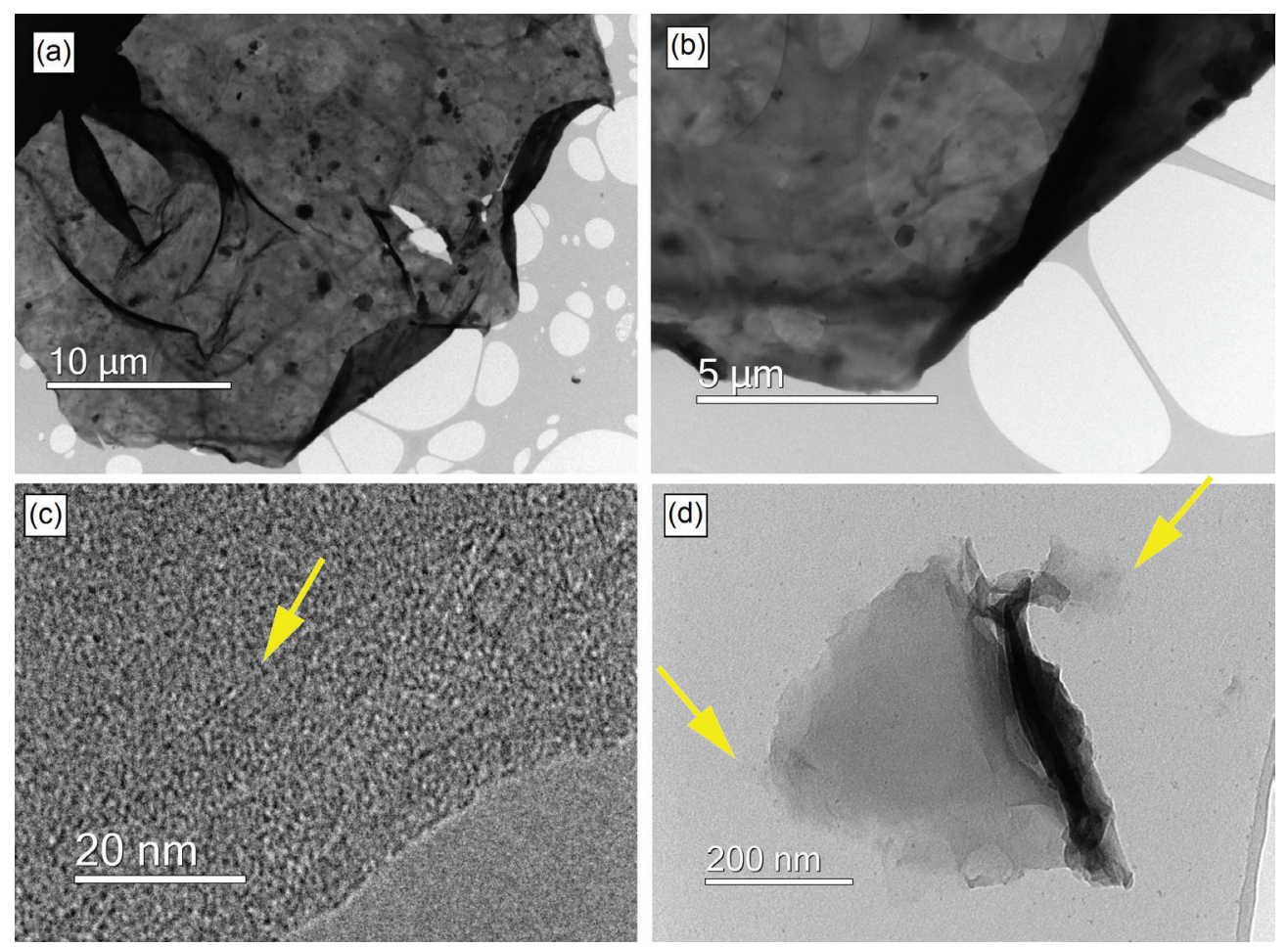

Figure 12. TEM images of sample GO + APTES. (a) A large sheet of functionalized graphene oxide; (b) same sheet at a higher magnification; (c) high resolution image of the edge of the sheet in (a) showing individual layers as the one indicated by the arrow; (d) a smaller sheet of functionalized graphene oxide, with amorphous material visible (indicated by the arrows). 
the pGO, albeit they still show a very high aspect ratio. Smaller sheets are also present in this sample, and one is shown (Figure 12d). Here, it is also possible to observe the presence of distinct layers, which appear to fold on themselves in the leftmost part of the sheet. Its edges are not as sharp as in sample pGO, and one can see a thin layer of an amorphous material, indicated by the arrows, covering parts of the edge.

\section{Conclusions}

By carefully controlling the parameters used in the functionalization of graphene oxide, and reacting it with (3-aminopropyl) triethoxysilane under mild conditions and at low concentration, we were able to obtain a GO-APTES hybrid that preserves the morphological characteristics of the parent GO, including its high aspect ratio and two-dimensionality, while including $\mathrm{SiOH}$ and $\mathrm{NH}_{2}$ functional groups on its surface. From the data revealed by XPS, FTIR and potentiometric titration and TGA, it was evidenced that the reaction involves both the amine and the silane groups of the APTES, reacting with different oxygen-containing groups present at the surface of the GO. The hybrid described in this work can be exfoliated and forms stable dispersions in water, unlike GO-APTES hybrids synthesized under harsher conditions and larger concentrations of APTES. The material obtained is promising for application as additive in polymeric matrices.

\section{Acknowledgments}

This work was supported by the Brazilian Nanocarbon Institute, the SisNano/MCTIC Program, the Brazilian agencies CNPq, CAPES, FAPEMIG and CNEN. The authors would like to thank the UFMG Microscopy Centre team for the electronic microscopy images, the Nanostructured Soft Materials Laboratory at LNNano/ CNPEM for XPS measurements, Dr Pedro Lana from CDTN for valuable XPS discussion and Dr Jefferson Nascimento and Ms Juliana Xavier from CDTN for carrying out the TGA analyses and helping in their discussion.

\section{References}

1. Geim, A. K.; Science 2009, 324, 1530.

2. Lee, C.; Wei, X.; Kysar, J. W.; Hone, J.; Science 2008, 321, 385.

3. Kuila, T.; Bose, S.; Mishra, A. K.; Khanra, P.; Kim, N. H.; Lee, J. H.; Prog. Mater. Sci. 2012, 57, 1061.

4. Dhand, V.; Rhee, K. Y.; Kim, H. J.; Jung, D. H.; J. Nanomater. 2013, 2013, ID 763953.
5. Yi, M.; Shen, Z.; J. Mater. Chem. A 2015, 3, 11700.

6. Park, S.; Ruoff, R. S.; Nat. Nanotechnol. 2009, 4, 217.

7. Dreyer, D. R.; Park, S.; Bielawski, C. W.; Ruoff, R. S.; Chem. Soc. Rev. 2010, 39, 228.

8. Haeri, S. Z.; Ramezanzadeh, B.; Asghari, M.; J. Colloid Interface Sci. 2017, 493, 111.

9. Wan, Y. J.; Gong, L. X.; Tang, L. C.; Wu, L. B.; Jiang, J. X.; Composites, Part A 2014, 64, 79.

10. Kuilla, T.; Bhadra, S.; Yao, D.; Kim, N. H.; Bose, S.; Lee, J. H.; Prog. Polym. Sci. 2010, 35, 1350.

11. Hu, L.; Jiang, P.; Zhang, P.; Bian, G.; Sheng, S.; Huang, M.; Bao, Y.; Xia, J.; J. Mater. Sci. 2016, 51, 8296.

12. Saravanan, N.; Rajasekar, R.; Mahalakshmi, S.; Sathishkumar, T. P.; Sasikumar, K.; Sahoo, S.; J. Reinf. Plast. Compos. 2014, $33,1158$.

13. Xia, H.; Zhang, X.; Shi, Z.; Zhao, C.; Li, Y.; Wang, J.; Qiao, G.; Mater. Sci. Eng., A 2015, 639, 29.

14. Bartolucci, S. F.; Paras, J.; Rafiee, M. A.; Rafiee, J.; Lee, S.; Kapoor, D.; Koratkar, N.; Mater. Sci. Eng., A 2011, 528, 7933.

15. Huang, X.; Qi, X.; Boey, F.; Zhang, H.; Chem. Soc. Rev. 2012, $41,666$.

16. Li, Z.; Wang, R.; Young, R. J.; Deng, L.; Yang, F.; Hao, L.; Jiao, W.; Liu, W.; Polymer 2013, 54, 6437.

17. Wang, X.; Xing, W.; Zhang, P.; Song, L.; Yang, H.; Hu, Y.; Compos. Sci. Technol. 2012, 72, 737.

18. Yang, H.; Li, F.; Shan, C.; Han, D.; Zhang, Q.; Niu, L.; Ivaska, A.; J. Mater. Chem. 2009, 19, 4632.

19. Lei, L.; Xia, Z.; Zhang, L.; Zhang, Y.; Zhong, L.; Prog. Org. Coat. 2016, 97, 19.

20. Ramezanzadeh, B.; Ahmadi, A.; Mahdavian, M.; Corros. Sci. 2016, 109, 182.

21. Sun, W.; Wang, L.; Wu, T.; Wang, M.; Yang, Z.; Pan, Y.; Liu, G.; Chem. Mater. 2015, 27, 2367.

22. Pourhashem, S.; Rashidi, A.; Vaezi, M. R.; Bagherzadeh, M. R.; Surf. Coat. Technol. 2017, 317, 1.

23. Rana, S.; Jonnalagadda, S. B.; Catal. Commun. 2017, 92, 31.

24. da Silva, C. T. P.; Monteiro, J. P.; Radovanovic, E.; Girotto, E. M.; Sens. Actuators, B 2014, 191, 152.

25. Kim, Y.; Park, H.; Kim, B.; High Perform. Polym. 2015, 27 , 886.

26. Guo, Z.; Wang, S.; Wang, G.; Niu, Z.; Yang, J.; Wu, W.; Carbon 2014, 76, 203.

27. CasaXPS Processing Software, version 2.3.19; Casa Software Ltd., Teignmouth, UK, 2018.

28. Pacheco, F. G.; Cotta, A. A. C.; Gorgulho, H. F.; Santos, A. P.; Macedo, W. A. A.; Furtado, C. A.; Appl. Surf. Sci. 2015, 357, 1015.

29. Wang, S.; Chia, P. J.; Chua, L. L.; Zhao, L. H.; Png, R. Q.; Sivaramakrishnan, S.; Zhou, M.; Goh, R. G. S.; Friend, R. H.; Wee, A. T. S.; Ho, P. K. H.; Adv. Mater. 2008, 20, 3440.

30. Abel, M.; Rattana, A.; Watts, J. F.; Langmuir 2000, 16, 6510. 
31. Graf, N.; Yegen, E.; Gross, T.; Lippitz, A.; Weigel, W.; Krakert, S.; Terfort, A.; Unger, W. E. S.; Surf. Sci. 2009, 603, 2849.

32. Gulino, A.; Anal. Bioanal. Chem. 2013, 405, 1479.

33. Gomes, G.; Gracielle, L.; Andrade, F.; Fernandes, M.; Wellington, C.; Lana, P.; Dawidson, G.; Gomes, A.; Coutinho, M.; Augusto, W.; Macedo, D. A.; Jose, M.; Edésia, N.; Barros, M.; J. Mater. Sci.: Mater. Med. 2018, 29, 130.

34. Ţucureanu, V.; Matei, A.; Avram, A. M.; Crit. Rev. Anal. Chem. 2016, 46, 502.

35. Kim, U. J.; Furtado, C. A.; Liu, X.; Chen, G.; Eklund, P. C.; J. Am. Chem. Soc. 2005, 127, 15437.

36. Kim, U. J.; Liu, X. M.; Furtado, C. A.; Chen, G.; Saito, R.; Jiang, J.; Dresselhaus, M. S.; Eklund, P. C.; Phys. Rev. Lett. 2005, 95, 157402.

37. Chen, L.; Jin, H.; Xu, Z.; Shan, M.; Tian, X.; Yang, C.; Wang, Z.; Cheng, B.; Mater. Chem. Phys. 2014, 145, 186.

38. Eaton, P.; Holmes, P.; Yarwood, J.; J. Appl. Polym. Sci. 2001, 82, 2016.

39. Kim, J.; Seidler, P.; Wan, L. S.; Fill, C.; J. Colloid Interface Sci. 2009, 329, 114.
40. Orth, E. S.; Ferreira, J. G. L.; Fonsaca, J. E. S.; Blaskievicz, S. F.; Domingues, S. H.; Dasgupta, A.; Terrones, M.; Zarbin, A. J. G.; J. Colloid Interface Sci. 2016, 467, 239.

41. Gorgulho, H. F.; Mesquita, J. P.; Gonçalves, F.; Pereira, M. F. R.; Figueiredo, J. L.; Carbon 2008, 46, 1544.

42. de Mesquita, J. P.; Martelli, P. B.; Gorgulho, H. F.; J. Braz. Chem. Soc. 2006, 17, 1133.

43. Vieira, H. S.; Andrada, D. M.; Mendonça, R.; Santos, A. P.; Martins, M. D.; Macedo, W. A. A.; Gorgulho, H. F.; Pimenta, L. P. S.; Moreira, R. L.; Jorio, A.; Pimenta, M. A.; Furtado, C. A.; J. Nanosci. Nanotechnol. 2007, 7, 3421.

44. Ganguly, A.; Sharma, S.; Papakonstantinou, P.; Hamilton, J.; J. Phys. Chem. C 2011, 115, 17009.

45. Zielke, U.; Hüttinger, K. J.; Hoffman, W. P.; Carbon 1996, 34 , 983.

46. Figueiredo, J.; Pereira, M. F.; Freitas, M. M.; Órfão, J. J.; Carbon 1999, 37, 1379.

47. Boehm, H.-P.; Diehl, E.; Heck, W.; Sappok, R.; Angew. Chem. 1964, 76, 742 . 